TITLE:

Necessary and Sufficient Numbers of Cards for Sharing Secret Keys on Hierarchical Groups (New Developments of Theory of Computation and Algorithms)

$\operatorname{AUTHOR}(S)$ :

Mizuki, Takaaki; Nishizeki, Takao

CITATION:

Mizuki, Takaaki ... [et al]. Necessary and Sufficient Numbers of Cards for Sharing Secret Keys on Hierarchical Groups (New Developments of Theory of Computation and Algorithms). 数理解析研究所講究録 2001, 1205: 13-18

ISSUE DATE:

2001-05

URL:

http://hdl.handle.net/2433/40993

RIGHT: 


\title{
Necessary and Sufficient Numbers of Cards for Sharing Secret Keys on Hierarchical Groups
}

\author{
東北大学大学院情報科学研究科＼cjkstart水木敬明 1 （Takaaki Mizuki） \\ 西関隆夫（Takao Nishizeki） \\ Graduate School of Information Sciences, \\ Tohoku University
}

\begin{abstract}
Suppose that there are players in two hierarchical groups and a computationally unlimited eavesdropper. Using a random deal of cards, a player in the higher group wishes to send a one-bit message information-theoretically securely either to all the players in her group or to all the players in the two groups. This can be done by the so-called 2-level key set protocol. In this paper we give a necessary and sufficient condition for the 2-level key set protocol to succeed.
\end{abstract}

\section{Introduction}

Suppose that there are $k(\geq 2)$ players $P_{1}, P_{2}, \cdots, P_{k}$ and a passive eavesdropper, Eve, whose computational power is unlimited. Consider a graph called a key exchange graph, in which each vertex $i$ represents a player $P_{i}$ and each edge $(i, j)$ joining vertices $i$ and $j$ represents a pair of players $P_{i}$ and $P_{j}$ sharing a one-bit secret key $r_{i j} \in\{0,1\}$ that is information-theoretically secure against the eavesdropper Eve. Refer to [6] for the graph-theoretic terminology. A connected graph having no cycle is called a tree. If the key exchange graph is a tree, then an arbitrary player can send a one-bit message $m \in\{0,1\}$ to all the players information-theoretically securely as follows: the player sends the message $m$ to the rest of the players along the tree; when player $P_{i}$ sends $m$ to player $P_{j}$ along an edge $(i, j)$ of the tree, $P_{i}$ computes the exclusive-or $m \oplus r_{i j}$ of $m$ and $r_{i j}$ and sends it to $P_{j}$, and $P_{j}$ obtains $m$ by computing $\left(m \oplus r_{i j}\right) \oplus r_{i j}$.

For $k=2$, Fischer et al. give a protocol using a random deal of cards to connect the two players $P_{1}$ and $P_{2}$ with an edge, that is, to form a tree on the two players [1]. (A random deal of cards will be formally described in Section 2.1.) Fischer and Wright extend this protocol to form a tree for any $k \geq 2$; they formalize a class of protocols called the "key set protocol," the definition of which will be given in Section $2.2[2,5]$. They also give a sufficient condition on the numbers of cards for the "key set protocol" to always form a tree. Mizuki et al. give a simple necessary and sufficient condition on the numbers of cards for the "key set protocol" to always form a tree [8].

On the other hand, Yoshikawa et al. consider the following more general problem [9]. Suppose that the $k$ players are partitioned into two hierarchical groups, which are represented as $V_{1}$ and $V_{2}$, where $V_{1} \cup V_{2}=\{1,2, \cdots, k\}$ and $V_{1} \cap V_{2}=\emptyset$. In the hierarchy, the group $V_{1}$ is assumed to be higher than the group $V_{2}$. Yoshikawa et al. wish to form, as a key exchange graph, a tree $T$ such that the subgraph $T_{1}$ of $T$ induced by $V_{1}$ is also a tree. Such a tree is called a 2-level tree (for the hierarchy). Once a 2-level tree $T$ is formed, any player in the higher group $V_{1}$ can send a one-bit message $m$ either to all the players in $V_{1}$ or to all the players in $V_{1} \cup V_{2}$, because both $T_{1}$ and $T$ are connected. Yoshikawa et al. modify the "key set protocol" in $[2,5]$ so that their protocol, called a "2-level protocol," forms a 2-level tree; the formal definition of the "2-level protocol" will be given in Section 2.3. They give a sufficient condition on the numbers

\footnotetext{
${ }^{1}$ PRESTO, JST
} 
of cards for the "2-level protocol" to always form a 2-level tree. However, their condition is not a necessary one, and hence it has been an open problem to obtain a necessary and sufficient condition.

In this paper, we give a necessary and sufficient condition on the numbers of cards for the "2-level protocol" to always form a 2-level tree, and hence close the open problem. Using our necessary and sufficient condition, one can easily know the minimum number of cards needed to form a 2-level tree.

\section{Preliminaries}

We first formally describe a random deal of cards in Section 2.1, then explain the "key set protocol" in Section 2.2, and finally explain the "2-level protocol" in Section 2.3.

\subsection{Random Deal of Cards}

In this subsection we formally describe a random deal of cards [4].

Let $C$ be a set of $d$ distinct cards which are numbered from 1 to $d$. All cards in $C$ are randomly dealt to players $P_{1}, P_{2}, \cdots, P_{k}$ and an eavesdropper Eve. We call a set of cards dealt to a player or Eve a hand. Let $C_{i} \subseteq C$ be $P_{i}$ 's hand for each $1 \leq i \leq k$, and let $C_{e} \subseteq C$ be Eve's hand. We denote this deal by $\mathcal{C}=\left(C_{1}, C_{2}, \cdots, C_{k} ; C_{e}\right)$. Clearly $\left\{C_{1}, C_{2}, \cdots, C_{k}, C_{e}\right\}$ is a partition of set $C$. We write $c_{i}=\left|C_{i}\right|$ for each $1 \leq i \leq k$ and $c_{e}=\left|C_{e}\right|$, where $|A|$ denotes the cardinality of a set $A$. Note that $c_{1}, c_{2}, \cdots, c_{k}$ and $c_{e}$ are the sizes of hands held by $P_{1}, P_{2}, \cdots, P_{k}$ and Eve respectively, and that $d=\sum_{i=1}^{k} c_{i}+c_{e}$. We call $\gamma=\left(c_{1}, c_{2}, \cdots, c_{k} ; c_{e}\right)$ the signature of $\operatorname{deal} \mathcal{C}$. The set $C$ and the signature $\gamma$ are public to all the players and even to Eve, but the cards in the hand of a player or Eve are private to herself, as in the case of usual card games.

Using a random deal of cards, a protocol can make several pairs of players share a one-bit secret key, as we will explain in the succeeding subsection. A reasonable situation in which such a protocol is practically required is discussed in $[3,5]$, and also the reason why we deal cards even to Eve is found there.

\subsection{Key Set Protocol}

In this subsection we explain the "key set protocol" formalized in $[2,5]$.

We first define some terms. A key set $K=\{x, y\}$ consists of two cards $x$ and $y$, one in $C_{i}$, the other in $C_{j}$ with $i \neq j$, say $x \in C_{i}$ and $y \in C_{j}$. We say that a key set $K=\{x, y\}$ is opaque if $1 \leq i, j \leq k$ and Eve cannot determine whether $x \in C_{i}$ or $x \in C_{j}$ with probability greater than $1 / 2$. Note that both players $P_{i}$ and $P_{j}$ know that $x \in C_{i}$ and $y \in C_{j}$. If $K$ is an opaque key set, then $P_{i}$ and $P_{j}$ can share a one-bit secret key $r_{i j} \in\{0,1\}$, using the following rule agreed on before starting a protocol: $r_{i j}=0$ if $x>y ; r_{i j}=1$, otherwise. Since Eve cannot determine whether $r_{i j}=0$ or $r_{i j}=1$ with probability greater than $1 / 2$, the secret key $r_{i j}$ is information-theoretically secure. We say that a card $x$ is discarded if all the players agree that $x$ has been removed from someone's hand, that is, $x \notin\left(\bigcup_{i=1}^{k} C_{i}\right) \cup C_{e}$. We say that a player $P_{i}$ drops out of the protocol if she no longer participates in the protocol. We denote by $V$ the set of indices $i$ of all the players $P_{i}$ remaining in the protocol. Note that $V=\{1,2, \cdots, k\}$ before starting a protocol.

The "key set protocol" has the following four steps. 
1. Choose a player $P_{s}, s \in V$, as a proposer by a certain procedure.

2. The proposer $P_{s}$ determines in mind two cards $x, y$. The cards are randomly picked so that $x$ is in her hand and $y$ is not in her hand, i.e. $x \in C_{s}$ and $y \in\left(\bigcup_{i \in V-\{s\}} C_{i}\right) \cup C_{e}$. Then $P_{s}$ proposes $K=\{x, y\}$ as a key set to all the players. (The key set is proposed just as a set. Actually it is sorted in some order, for example in ascending order, so Eve learns nothing about which card belongs to $C_{s}$ unless Eve holds $y$.)

3. If there exists a player $P_{t}$ holding $y$, then $P_{t}$ accepts $K$. Since $K$ is an opaque key set, $P_{s}$ and $P_{t}$ can share a one-bit secret key $r_{s t}$ that is information-theoretically secure from Eve. (In this case an edge $(s, t)$ is added to the key exchange graph.) Both cards $x$ and $y$ are discarded. Let $P_{i}$ be either $P_{s}$ or $P_{t}$ that holds the smaller hand; if $P_{s}$ and $P_{t}$ hold hands of the same size, let $P_{i}$ be the proposer $P_{s}$. $P_{i}$ discards all her cards and drops out of the protocol. Set $V:=V-\{i\}$. Return to step 1 .

4. If there exists no player holding $y$, that is, Eve holds $y$, then both cards $x$ and $y$ are discarded. Return to step 1. (In this case no new edge is added to the key exchange graph.)

These steps 1-4 are repeated until either exactly one player remains in the protocol or there are not enough cards left to complete step 2 even if two or more players remain. In the first case the key exchange graph becomes a tree. In the second case the key exchange graph does not become a connected graph and hence does not become a tree.

Considering various procedures for choosing a proposer $P_{s}$ in step 1 , we obtain the class of key set protocols.

We say that a key set protocol works for a signature $\gamma$ if the protocol always forms a tree as a key exchange graph for any deal $\mathcal{C}$ having the signature $\gamma$ and for any random selection of cards $x$ and $y$ in step 2 . Let $k \geq 2$ and $\gamma=\left(c_{1}, c_{2}, \cdots, c_{k} ; c_{e}\right)$. Let $W$ be the set of all signatures for each of which there is a key set protocol working, and let $L$ be the set of all signatures for each of which there is no key set protocol working. A simple necessary and sufficient condition for $\gamma \in W$ has been known $[2,8]$. Furthermore, a characterization of "optimal" key set protocols is given in [7].

\subsection{2-Level Protocol}

In this subsection we explain the "2-level protocol" given in [9].

Suppose that there are two hierarchical groups $V_{1}$ and $V_{2}$. The "2-level protocol" forms a 2-level tree, whose subgraph induced by $V_{1}$ is connected. The "2-level protocol" forms a 2-level tree in which every vertex in $V_{2}$ has degree one, that is, every vertex in $V_{2}$ is a leaf. The "2-level protocol" is obtained by slightly modifying steps 1 and 3 in the key set protocol, as follows: in step 1 , a player in $V_{1}$ is always chosen as a proposer $P_{s}$; and in step 3 , whenever card $y$ is held by a player $P_{t}$ in $V_{2}, P_{t}$ drops out of the protocol even if $P_{t}$ holds the larger hand than $P_{s}$. Thus the "2-level protocol" has the following four steps.

1. Choose a player $P_{s}, s \in V_{1}$, as a proposer by a certain procedure.

2. The proposer $P_{s}$ randomly determines in mind two cards $x, y$ so that $x$ is in her hand and $y$ is not in her hand. Then $P_{s}$ proposes $K=\{x, y\}$ as a key set to all the players. 
3. If there exists a player $P_{t}$ holding $y$, then $P_{s}$ and $P_{t}$ can share a one-bit secret key $r_{s t}$. Both cards $x$ and $y$ are discarded.

(a) If $t \in V_{1}$, then let $P_{i}$ be either $P_{s}$ or $P_{t}$ that holds the smaller hand; when $P_{s}$ and $P_{t}$ hold hands of the same size, let $P_{i}$ be the proposer $P_{s} . P_{i}$ discards all her cards and drops out of the protocol. Set $V_{1}:=V_{1}-\{i\}$. Return to step 1 .

(b) If $t \in V_{2}$, then $P_{t}$ discards all her cards and drops out of the protocol. Set $V_{2}:=$ $V_{2}-\{t\}$. Return to step 1.

4. If there exists no player holding $y$, that is, Eve holds $y$, then both cards $x$ and $y$ are discarded. Return to step 1.

These steps 1-4 are repeated until either exactly one player in $V_{1}$ remains in the protocol or there are not enough cards left to complete step 2 even if two or more players remain. In the first case the key exchange graph becomes a 2-level tree, in which every vertex in $V_{2}$ has degree one. In the second case the key exchange graph does not become a 2-level tree.

Considering various procedures for choosing a proposer $P_{s}$ in step 1 , we obtain the class of 2-level protocols.

Without loss of generality one may assume that $V_{1}=\left\{1,2, \cdots, k_{1}\right\}$ and $V_{2}=\left\{k_{1}+\right.$ $\left.1, k_{1}+2, \cdots, k_{1}+k_{2}\right\}$ where $k=k_{1}+k_{2}$. One may assume that all the players in $V_{2}$ hold at least one card, i.e. $c_{i} \geq 1$ for all $i, k_{1}+1 \leq i \leq k_{1}+k_{2}$. Once an edge is connected to a player in $V_{2}$ during the execution of any 2-level protocol, the player in $V_{2}$ necessarily drops out of the protocol. Therefore any player in $V_{2}$ does not need two or more cards. More precisely, there is a 2-level protocol which always forms a 2-level tree for $\gamma=$ $\left(c_{1}, c_{2}, \cdots, c_{k_{1}}, c_{k_{1}+1}, c_{k_{1}+2}, \cdots, c_{k_{1}+k_{2}} ; c_{e}\right)$ if and only if there is a 2-level protocol which always forms a 2-level tree for $\gamma=\left(c_{1}, c_{2}, \cdots, c_{k_{1}}, 1,1, \cdots, 1 ; c_{e}\right)$. We thus use a 2-level signature $\alpha=$ $\left(c_{1}, c_{2}, \cdots, c_{k_{1}} ; k_{2} ; c_{e}\right)$ to represent a signature $\gamma=\left(c_{1}, c_{2}, \cdots, c_{k_{1}}, c_{k_{1}+1}, c_{k_{1}+2}, \cdots, c_{k_{1}+k_{2}} ; c_{e}\right)$. Remember that $k_{2}$ is the number of players in $V_{2}$.

We say that a 2-level protocol works for a 2-level signature $\alpha$ if the protocol always forms a 2-level tree as a key exchange graph for any deal $\mathcal{C}$ having the 2-level signature $\alpha$ and for any random selection of cards $x$ and $y$ in step 2. Let $k_{1} \geq 1, k_{1}+k_{2} \geq 2$, and $\alpha=\left(c_{1}, c_{2}, \cdots, c_{k_{1}} ; k_{2} ; c_{e}\right)$. One may assume without loss of generality that $c_{1} \geq c_{2} \geq \cdots \geq c_{k_{1}}$. Let $W^{2}$ be the set of all 2-level signatures for each of which there is a 2-level protocol working, and let $L^{2}$ be the set of all 2-level signatures for each of which there is no 2-level protocol working.

We say that a player $P_{i}, i \in V_{1}$, is feasible in a 2-level signature $\alpha=\left(c_{1}, c_{2}, \cdots, c_{k_{1}} ; k_{2} ; c_{e}\right)$ if the following condition (1), (2) or (3) holds.

(1) $c_{i} \geq 2$.

(2) $k_{2}=0, c_{e}=0, c_{i}=1$ with $i=k_{1}$, and $c_{k_{1}-1} \geq 2$.

(3) $k_{1}=k_{2}=1, c_{e}=0$, and $c_{i}=1$ with $i=1$.

We define a mapping $g$ from the set of all 2-level signatures to $\left\{0,1,2, \cdots, k_{1}\right\}$, as follows: $g(\alpha)=i$ if $P_{i}$ is the feasible player in $\alpha$ with the smallest hand (ties are broken by selecting the player having the largest index); and $g(\alpha)=0$ if there is no feasible player. For example, if $\alpha=(9,9,8,6,5,3,2,2,1,1 ; 2 ; 2)$ as illustrated in Figure 1 , then $g(\alpha)=8$. We denote $g(\alpha)$ simply by $g$.

Yoshikawa et al. give a sufficient condition for $\alpha \in W^{2}$ as in the following Theorem 1. 


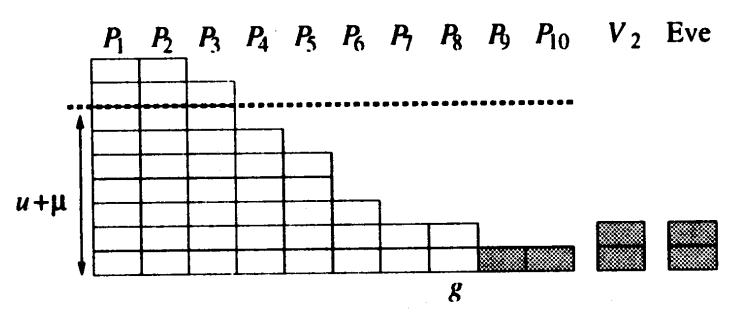

Figure 1: An illustration of $\alpha=(9,9,8,6,5,3,2,2,1,1 ; 2 ; 2)$.

Theorem 1 ([9]) Let $k_{1} \geq 1, k_{2} \geq 1$, and $c_{k_{1}} \geq 1$. If there exists $k_{0}$ such that $0 \leq k_{0} \leq k_{1}-1$ and $c_{k_{1}-k_{0}} \geq c_{e}+\left\lfloor\log _{2}\left(k_{1}-k_{0}\right)\right\rfloor+k_{0}+k_{2}$, then $\alpha \in W^{2}$.

They prove Theorem 1 by showing that the 2-level protocol choosing the player $\boldsymbol{P}_{\boldsymbol{g}}$ as a proposer works for any 2-level signature satisfying the condition in Theorem 1. However, their sufficient condition in Theorem 1 is not a necessary one. For example, the 2-level signature $\alpha=(9,9,8,6,5,3,2,2,1,1 ; 2 ; 2)$ above does not satisfy their sufficient condition in Theorem 1 , while it is actually in $W^{2}$ as we will see in Section 3 . Thus it has been an open problem to obtain a necessary and sufficient condition for $\alpha \in W^{2}$. This paper closes the open problem in Section 3 , that is, provides a necessary and sufficient condition for $\alpha \in W^{2}$.

\section{Main Results}

In this section we give a necessary and sufficient condition for $\alpha \in W^{2}$.

Our main result is the following Theorem 2 . Hereafter we define $B=\left\{i \mid c_{i}=2,1 \leq i \leq k_{1}\right\}$ and $b=\lfloor|B| / 2\rfloor$ for a 2-level signature $\alpha$.

Theorem 2 Let $k_{1} \geq 1, k_{2} \geq 1, c_{k_{1}} \geq 1$, and $g \geq 1$. Then $\alpha=\left(c_{1}, c_{2}, \cdots, c_{k_{1}} ; k_{2} ; c_{e}\right) \in W^{2}$ if and only if

$$
c_{1}-(u+\mu)+\sum_{i=2}^{k_{1}} \max \left\{c_{i}-(u+\mu), 0\right\} \geq g-2 \mu-1,
$$

where

$$
u=c_{e}+k_{1}+k_{2}-g
$$

and

$$
\mu=\max \left\{\min \left\{c_{3}-u, b\right\}, 0\right\} \text {. }
$$

Note that the third term in the left side of Eq. (1) is defined to be 0 when $k_{1}=1$, and that $\mu$ is defined to be 0 when $k_{1} \leq 2$.

Consider again $\alpha=(9,9,8,6,5,3,2,2,1,1 ; 2 ; 2)$ as an example. The 2-level signature $\alpha$ satisfies $k_{1}=10, k_{2}=2, c_{e}=2$ and $g=8$. Thus by Eq. (2) $u=6$. Note that $u$ is equal to the number of shaded rectangles in Figure 1 . Since $B=\{7,8\}, b=1$. Since $c_{3}=8, u=6$ and $b=1$, we have $\mu=1$ by Eq. (3). Thus $c_{1}-(u+\mu)+\sum_{i=2}^{k_{1}} \max \left\{c_{i}-(u+\mu), 0\right\}=$ $c_{1}-7+\sum_{i=2}^{10} \max \left\{c_{i}-7,0\right\}=5=g-2 \mu-1$. Therefore the 2-level signature $\alpha$ satisfies the condition (1) in Theorem 2, and hence $\alpha \in W^{2}$. Note that the left side of Eq. (1) is equal to the number of cards above the dotted line in Figure 1. 
From Theorem 2 we have the following Corollary 3, which provides a necessary and sufficient condition for $\alpha \in W^{2}$ under a natural assumption that all players in $V_{1}$ hold hands of the same size.

Corollary 3 Let $k_{1} \geq 1, k_{2} \geq 1, c_{k_{1}} \geq 1, g \geq 1$, and $c_{1}=c_{2}=\cdots=c_{k_{1}}$. Then $\alpha \in W^{2}$ if and only if

$$
c_{1} \geq \begin{cases}3 & \text { if } k_{1} \geq 4, k_{2}=1 \text { and } c_{e}=0 \\ c_{e}+k_{2} & \text { if } k_{1}=1 ; \text { and } \\ c_{e}+k_{2}+1 & \text { otherwise. }\end{cases}
$$

Theorem 1 obtained by Yoshikawa et al. [9] implies that a sufficient condition for $\alpha \in W^{2}$ is $c_{1} \geq c_{e}+k_{2}+\left\lfloor\log _{2} k_{1}\right\rfloor$ when $c_{1}=c_{2}=\cdots=c_{k_{1}}$. Thus our necessary and sufficient condition in Theorem 2 is much better than the sufficient condition in [9].

Due to the page limitation, we omit a proof of Theorem 2 in this extended abstract.

\section{References}

[1] M. J. Fischer, M. S. Paterson, and C. Rackoff, "Secret bit transmission using a random deal of cards," DIMACS Series in Discrete Mathematics and Theoretical Computer Science, AMS, vol. 2, pp. 173-181, 1991.

[2] M. J. Fischer and R. N. Wright, "An application of game-theoretic techniques to cryptography," DIMACS Series in Discrete Mathematics and Theoretical Computer Science, AMS, vol. 13, pp. 99-118, 1993.

[3] M. J. Fischer and R. N. Wright, "An efficient protocol for unconditionally secure secret key exchange," Proc. of the 4th Annual Symposium on Discrete Algorithms, pp. 475 483, 1993.

[4] M. J. Fischer and R. N. Wright, "Bounds on secret key exchange using a random deal of cards," J. Cryptology, vol. 9, pp. 71-99, 1996.

[5] M. J. Fischer and R. N. Wright, "Multiparty secret key exchange using a random deal of cards," Proc. CRYPTO '91, Lecture Notes in Computer Science, Springer-Verlag, vol. 576, pp. 141-155, 1992.

[6] F. Harary, "Graph Theory," Addison-Wesley, Reading, Mass., 1969.

[7] T. Mizuki, H. Shizuya, and T. Nishizeki, "Characterization of optimal key set protocols," Proc. IFIP TCS 2000, Lecture Notes in Computer Science, Springer-Verlag, vol. 1872, pp. 273-285, 2000.

[8] T. Mizuki, H. Shizuya, and T. Nishizeki, "Dealing necessary and sufficient numbers of cards for sharing a one-bit secret key," Proc. EUROCRYPT '99, Lecture Notes in Computer Science, Springer-Verlag, vol. 1592, pp. 389-401, 1999.

[9] R. Yoshikawa, S. Guo, K. Motegi, and Y. Igarashi, "Secret key exchange using random deals of cards on hierarchical structures," Proc. ISAAC 2000, Lecture Notes in Computer Science, Springer-Verlag, vol. 1969, pp. 290-301, 2000. 